\title{
Cuidar tiene rostro de mujer Ecofeminismo, Paradigma de la esperanza
}

Cecilia Ré *

\section{Resumen}

Este ensayo científico presenta la política de defensa de la vida encarnada en una triSofía: la Cuidadología, promocionando la salud, tanto la humana como la de los entornos, sinergizada con el Ecofeminismo, teoría y movimiento social que busca despatriarcalizar los sistemas. Amalgamadas, sintetizan una Cuidadosofía que significan la esperanza de alcanzar salud para todos, justicia social y verdadero desarrollo humano. La cultura del cuidado se renueva rescatando saberes ancestrales holísticos.

Palabras clave: $\quad$ Cuidadosofia - Ecofeminismo - Holismo - Sistema de Salud 


\title{
Caring has a woman's face Ecofeminism, Paradigm of Hope
}

\author{
Cecilia Ré *
}

\begin{abstract}
This scientific essay presents the policy of defense of life embodied in a tri sofia: Careology, promoting health, both human and environment, synergized with Ecofeminism, theory and social movement that seeks to depatriarchalize systems. Amalgamated, synthesize a Careosophy that mean the hope of achieving health for all, social justice, and true human development. The culture of care is renewed by rescuing holistic ancestral knowledge.
\end{abstract}

Keywords: Philosophy of care - Ecofeminism - Holism - Health System 


\section{Introducción}

"La salud se produce cuando se devuelve a la gente el poder para efectuar las transformaciones necesarias que aseguren un buen vivir y se reduzcan las causas que atentan contra la salud y el bienestar".

Carta de Ottawa, 1986

En un mundo violento, esquilmado, contaminado, la salud es más que nunca, un asunto de vida o muerte, dependiendo de un hilo, de las condiciones medioambientales.

Paradigma o modelo es un molde, una estructura, un arquetipo que sirve de inspiración, de referencia, juzgado como un valor o un bien y, entonces, tratamos de imitarlo o de reproducirlo.

Con esta intención de parámetro deseable, lo analizamos intelectualmente, estableciendo un esquema teórico, considerando sus partes operativas- subsistemas- y su totalidad funcional- sistema.

Un modelo o Sistema de Atención Médica o de Enfermería debería ser el reflejo de procesos históricos, económicos y culturales madurativos, involucrando la filosofía de vida de la comunidad, reflejada en los responsables de establecerlo. La fuerza de la visión incide en la planificación, ejecución y evaluación de las prácticas culturales profesionales efectuadas por el equipo para la salud. Su adecuación con la filosofía de la sociedad y sus prácticas de mantenimiento y restablecimiento de la salud, son las que aseguran su éxito.

Para desarrollar la salud comunitaria del siglo XXI con eficacia, la Enfermería, el equipo de salud, las comunidades científicas y políticas mundiales necesitan alfabetizarse en indicadores que sostienen a las nociones salud y bienestar.

El Ecofeminismo necesita ser trasplantado en el corazón curricular, particularmente en el de la Enfermería, con cuidados dirigidos a preservar la vida, manteniendo la salud, ubicados en la Atención Primaria de la Salud (APS). Este nivel abraza la salubridad de los entornos, principalmente el natural.

\section{Modelo médico hegemónico}

Todo modelo, organización, sistema debería responder a significados y sentidos sociales representativos de esta, dejando de ser un injerto extrapolado de otras culturas: inspiración posible, pero nunca reproducción acrítica ni parcial ni total.

La posibilidad del error, de la incompletud, del engaño: “...incluso este aparente método riguroso ha sufrido: desde la falsificación de resultados, a la no publicación de los ensayos clínicos negativos, o la publicación sin declarar los intereses económicos que han pagado la investigación. Mostrando una vez más una ciencia médica desvirtuada en función del mercado" (Antequera, 2013) por lo que copiar no es aconsejable. 
El modelo de atención de la salud o, mejor dicho, de servicio para el ciudadano enfermo, o en vías de sufrir una situación de cronicidad, muestra un marcado deterioro económico y de eficacia.

Este paradigma de medicina cientificista, positivista, mercantilista pierde poder y entra en tensión con otros emergentes que cuentan con mayor grado de aceptación por parte de la comunidad y de otros profesionales para el cuidado de la salud, como la Enfermería.

Espinosa Brito (2013) retrata los rasgos básicos del modelo médico hegemónico:

- biologismo y concepción metodológica-positivista,

- dominio de un marco teórico-ideológico-evolucionista y cartesiano,

- ahistoricidad, asocialidad, individualismo,

- la salud y la enfermedad como mercancías en términos directos o indirectos,

- eficacia pragmática,

- dominio de una orientación discursiva asistencial,

- concepción de la enfermedad como ruptura, diferencia,

- práctica curativa basada en la eliminación del síntoma,

- relación asimétrica médico-paciente,

- subordinación técnica y social del paciente,

- concepción del paciente como ignorante o portador de un saber equivocado,

- el paciente como responsable de su enfermedad (culpar a la víctima),

- el paciente como consumidor pasivo,

- exclusión del paciente del saber médico,

- escasa aplicación de actividades preventivas y dominio de una prevención no estructural,

- no legitimación científica de otros saberes,

- profesión formalizada, identificación ideológica con la racionalidad científica y tendencia a la escisión teórico-práctica.

Respecto a las actividades de promoción y de mantenimiento de la salud que originan la prevención de la enfermedad o de accidentes, centrándose en un supuesto curar, tecnologizando y medicalizando, con consecuencias indeseadas y efectos secundarios, algunos de ellos, graves.

Ejerce un control normado, impuesto por política, moral y ley, sobre el paciente y sobre los otros profesionales miembros del equipo para la salud, haciendo depender sus voluntades y saberes, concentrando poder económico por esta sujeción ocupacional, prioritariamente hospitalaria.

\section{Modelo emergente de autocuidado holístico}

Nacido del alma popular, suele ser ancestral, recogiendo sabiduría transgeneracional e intercontinental, con la particularidad de reducir los altos costos de esta medicina anglosajona y europea implantadas en suelo latinoamericano. 
Contrapuestos al anterior, aparecen ideas de Promoción de la salud y del bienestar, fruto del autocuidado con raíz femenina, relacionadas con la Enfermería, ciencia que le correspondería autoidentificarse como Cuidadología (Brito Brito, 2017; RÉ, 2016) tendiendo a la preservación de la autonomía individual y colectiva.

Las prácticas sanitarias de autocuidado se identifican con decisiones éticas individuales, realizadas en beneficio de su propia salud, basadas en informaciones derivadas de la educación y consejería para la salud.

La sumatoria de estas prácticas favorables, positivas y acertadas resultan en la adopción de un estilo de vida saludable con sentido para la persona. Se centran en la prevención de riesgos, en la prolongación de la sensación de bienestar y en la gestión de pequeñas dolencias cotidianas evitando que se intensifiquen y se agraven en el tiempo.

Gravitan en conocimientos sobre el buen vivir y el vivir bien:

- Alimentación y vestido.

- Medidas higiénicas.

- Manejo del estrés.

- Habilidades para establecer relaciones sociales interpersonales saludables.

- Equilibrio actividad física y reposo.

- Herbolario.

- Comportamientos sexuales seguros.

- Recreación y manejo del tiempo libre.

- Búsqueda de información y de concejo oportuno.

- Gestión del confort

- Autoestima, belleza y estética.

- Reconocimiento de la dependencia de la vida de la naturaleza para asegurar la supervivencia humana y de las otras especies.

Autocuidado y aprendizaje integral forman un binomio favorecido por la cooperación y la coordinación de grupos de apoyo, las redes tecnológicas y sociales, destacando el voluntariado sin edad.

El cuidado holístico respeta las capacidades, los conocimientos, los ritmos de la persona, sus creencias y valores, así como sus preferencias y su potencialidad. Considera el entorno y el apoyo social del que dispone. Vela por obtener alternativas de solución que satisfagan cierto nivel de dependencia.

La cultura del cuidado holístico con inspiración central en el Ecofeminismo permite pensar una sociedad sostenible, a través de valores como la reciprocidad, la cooperación y la complementariedad. En este contexto, Jean Watson teoriza que "la enfermera es la llamada a ayudar al paciente a aumentar la armonía entre la mente, cuerpo y el alma, para generar procesos de autoconocimientos" (Veliz Rojas, 2017).

\section{Barreras para el modelo emergente}

La sociedad contemporánea posee sistemas organizativos sociales erróneos: 
- la edad parvularia y la avanzada, la discapacidad necesitan de apoyos suplementarios humanizados y no solo de dispositivos técnicos,

- la ignorancia multidimensional sobre el humano, la realidad, la salud, la felicidad, - la desprotección del individuo, la familia y de la maternidad, en una era de promoción de derechos donde los organismos políticos no están a la altura,

- la discriminación hacia la mujer,

- el trabajo organizado en jornada extendida y fuera del hogar,

- las exigencias de trabajar más allá de los 55 años,

- las exigencias productivas impuestas a la Naturaleza sin posibilidad de renuevo,

- la industria alimentaria y farmacéutica centradas en el dinero,

- la insuficiente inversión en recursos humanos para la salud y la educación atentan contra este derecho,

- un sistema educativo que no abre sus puertas a los avances de la Física Cuántica sobre la realidad multiversa, a formas cualitativas de producir conocimiento, etc.

\section{Políticas integrales sanitarias}

Un modelo de servicio para la salud ciudadana cobra vida mediante la gente que lo operativiza con sus prácticas sociales ejecutadas dentro de los muros de una institución, que forma parte del sistema.

Cada práctica preventiva debería estar regulada por algún cuerpo normativo que vele por el trato respetuoso, su cumplimiento, su duración - no menos de 16 horas diarias, un recurso humano multidisciplinario entrenado adecuadamente en Atención Primaria de la Salud Holística, considerando la protección de derechos ciudadanos, relevados de la Ética y sus ramas (Metaética, Bioética, Deontología, Moral).

El modelo de servicio para la salud debe pensarse en términos de promoción mediante cuidados que satisfacen absolutamente todas las necesidades humanas básicas, satisfactores que previenen la enfermedad y los accidentes, para luego centrarnos en curar y rehabilitar.

La política debería ser provida y abierta porque la medicina occidental moderna y contemporánea ha validado prácticas profesionales que subordinan los saberes preventivos tradicionales a intereses económicos que enferman al hombre yendo contra la naturaleza de este. Conocidos son los trabajos sobre la distorsión de resultados científicos y otras debilidades académicas (Schulz, 2003; Daros, 1998; Muñoz van den Eynde, 2019).

Estas prácticas médicas economicistas no comprenden a las necesidades humanas fundamentales para su existencia: están despojadas de una lógica de humanidad que preservan a la vida. Tamez Pérez (2012, pág.13) dice" no se puede dejar de mencionar la presión que ejercen sobre el médico las industrias farmacéuticas y de manufactura de dispositivos, los administradores de compañías que hoy ofrecen servicios de salud, a menudo sin base en evidencias científicas sólidas, para tratar de conseguir que el médico 
prescriba o utilice sus medicamentos o que solicite pruebas diagnósticas en situaciones no justificadas ".

El sistema sanitario, el económico y el político actúan como escindidos de los conocimientos que entornan a la Vida, los que sus representantes deberían poseer y a los que la Medicina debería haber confrontado, basados en su juramento hipocrático.

\section{Salud global}

Para mejorar la salud global del planeta se necesita elevar el nivel de conciencia sobre el valor del binomio Vida Humana - Naturaleza. Las inversiones y las actuaciones para la salud deberían atender a una rápida identificación, disminución y eliminación de los contaminantes físicos, químicos, biológicos y sociales que atentan contra cualquier vida.

Las practicas sanitarias y económicas deberían unirse y destinarse a proteger con urgencia la salud: "Los niños que nazcan hoy serán más vulnerables a la enfermedad y se convertirán en jóvenes y adultos con más lesiones médicas y menor esperanza de vida, concluye el informe 'The Lancet Countdown 2019" (Torices, 2019, en Las Provincias) pero la ciega economía produce 100 millones de barriles de petróleo por día (Cooper, 2018) en vez de apostar a energías limpias.

La organización Mundial para la Salud (2018) explica que "las pruebas son claras: la contaminación atmosférica tiene un efecto devastador en la salud infantil". El futuro de la salud comunitaria se presenta con amenazas, desafíos y oportunidades de cooperación para los profesionales biosanitarios y de restantes sectores sociales implicados en el bienestar humano.

Esta crisis permite que surjan los potenciales dormidos de perfiles profesionales, estructuras de conocimiento y arquitecturas para el compromiso subestimado por la condición de género, raza, religión, política o filosofía.

La salud comunitaria, mejor dicho, la sobrevivencia planetaria requiere de liderazgos políticos ciudadanos valientes, empoderados con valores y conocimientos como recursos sustanciales.

La creatividad es bienvenida en este grupo, pero la improvisación es inaceptable, pues son la inconciencia y la inconsistencia intelectual egocéntrica de los líderes las que nos han llevado al caos actual. Magnánimos y profundos cambios sociales son necesarios y la única forma de lograrlo es la comprensión de la complejidad con mirada de humanidad.

La colaboración interplanetaria para mejorar la salud de las presentes y futuras generaciones es necesaria, contemplando la preservación de la integrad de la naturaleza: el medio ambiente necesita cuidados amorosos tanto como las personas. 


\section{Ecofeminismo}

El paradigma masculino de la Medicina, autocatalogada de científica, jerarquizada en la cúspide de las disciplinas, ha rechazado y combatido formas tradicionales de cuidado y de cura, ejercida por mujeres. Existen variados estudios culturales que ejemplifican la tradición histórica femenina ligada a la sabiduría del cuidado de la salud, de la belleza, de la nutrición, tildadas de herbolarias, sanadoras, curanderas, etc. (Cuadrada, 2014; Massé, 2017; Iglesias Aparicio, 2003) demostrando una comprensión sobre la vida muy diferente de la postura masculina.

Este modelo preventivo se dirige a todas las personas, pero en especial a aquellas en circunstancias de vulnerabilidad económica, ofreciendo cuidado domiciliario, no o escasamente remunerado, aun en nuestros días. "A lo largo de toda la historia quienes se han ocupado de los cuerpos vulnerables son mujeres" dice Yayo Herrero (Cuneo, 2020) cuerpos enfermos, ancianos, discapacitados y de niños.

El patriarcado y el sistema capitalista neoliberal son factores de riesgo para la salud humana y planetaria, amenazando las vidas de las comunidades y la del ambiente natural del cual estas dependen. La "despatriarcalización" es un reto colectivo solidario e internacional.

El modelo médico hegemónico se sustenta en la filosofía de la medicina destinada a mantener la enfermedad: es un paradigma de atención de la enfermedad que necesita que se perpetúe. El punto de vista del modelo ecofeminista trata del cuidado holístico cuerpo-mente-espíritu y medio ambiente, por la promoción y mantenimiento de la salud.

Ecofeminismo es Sofía del Cuidado remitiendo a la sabiduría, conocimiento y arte del cuidado: Cuidadología, llamada Enfermería por estar subyugada para sostener el modelo de la enfermedad, enclaustrada en el Hospital.

El autocuidado holístico devuelve el control del individuo sobre su salud. El desafío de las cuidadologas es educar a la sociedad para que pase de una actitud pasiva de receptor de cuidados, a una activa y proactiva, de búsqueda de bienestar y de responsabilidad sobre su propia salud.

Este modelo integrativo nutrido de las terapias ancestrales orientales y autóctonas, dispensadas en el marco del contrato de la relación terapéutica, asegura trato digno, aceptación cultural y demostración científica de su efectividad, como ocurre en otros países, formando parte de las obras sociales (Dirección de Medicina Tradicional y Desarrollo Intercultural, 2017; Johannessen, 1998; Office fédéral de la santé publique, s/f; Didier, 2016; Moreira, 2019, Papadakis 2015).

\section{Conclusión}

La fusión Ecofeminismo, Cuidadologia, Cuidadosofia con un leitmotiv compartido, la Promoción de la Salud por el autocuidado holístico, es la respuesta al déficit en la salud poblacional y al organizacional internacional. 
El actual sistema médico hegemónico merma el derecho fundamental a la vida, por robo de la autonomía moral sobre su cuerpo, impuesto políticamente por los sistemas homogeneizados de la salud, la farmacéutica y la alimentaria, bajo el modelo económico capitalista- neoliberalista, centrados en el lucro.

Implantar esta fusión paradigmática con rostro de mujer en el sistema educativo, priorizando el clínico-terapéutico significa reganar esperanza y emprender los pasos hacia una salud para todos con prácticas de medicinas integrativas queridas por la gente. Permitirá el ejercicio pleno de la autonomía de elección del modelo sanitario que se quiere seguir

Esta trifilosofía es una oportunidad única para fortalecer la salud comunitaria y otorgar servicios pertinentes, eficaces, seguros y sentidos, con bajo costo material y humano, en términos de muertes innecesarias y prematuras.

Como bien dijo Lao Tse "Diez gramos de prevención equivalen a un kilogramo de curación". Es una rica oportunidad de alfabetización con nuevos conocimientos y prácticas, algunas de ellas con identidad nacional. Queda el desafío pendiente del mito hecho realidad.

\section{Referencias bibliográficas}

ANTEQUERA, M. d. C. (2013). Análisis desde el punto de vista feminista del rol de la medicina en el modelo de salud médico-hegemónico. VII Jornadas de Jóvenes Investigadores. Instituto de Investigaciones Gino Germani, Facultad de Ciencias Sociales, Universidad de Buenos Aires, Buenos Aires. Disponible en https://www.aacademica.org/000-076/191.pdf

BRITO BRITO, P. R. (2017). Cuidadología: pensamientos sobre el nombre de nuestra disciplina. ENE, Revista de Enfermería. v. 11, n. 2, ago 2017. Disponible en http://eneenfermeria.org/ojs/index.php/ENE/article/view/711/cuidadologia

COOPER, A. (2018). Ahora cerca de 100 millones de bpd, ¿cuándo alcanzará un pico la demanda de petróleo? Disponible en https://www.reuters.com/article/us-oildemand-peak/now-near-100-millionbpd-when-will-oil-demand-peakidUSKCN1M01TC

CUADRADA, C. (2014). Cuidado, curación, salud: saberes de mujeres. História: Questões \& Debates, Curitiba, n. 60, p. 229-253, jan./jun. 2014. Editora UFPR. Disponible en https://revistas.ufpr.br/historia/article/download/38288/23435

CUNEO, M. (2020). Ecofeminismo. Entrevista a Yayo Herrero. Disponible en https://www.elsaltodiario.com/ecofeminismo/entrevista-yayo-herrero-

econom\%C3\%ADa-tecnolog\%C3\%ADa-pol\%C3\%ADtica-sociedad-naturalezacuidados

DAROS, W. (1998). Introducción a la epistemología Popperiana. CONICET-CERIDER: Consejo Nacional de Investigaciones Científicas y Técnicas. Centro Regional de 
Investigación y Desarrollo de Rosario. Disponible en https://www.ucel.edu.ar/images/2018/PDF_UCEL/Libros_publicados/Filosofia/Intro duccin-a-la-filosofa-popperianaDAROS7.pdf

DIDIER, P. (2016). Médecine traditionnelle et Médecine intégrative à Madagascar : entre décisions internales et applications locales. Disponible en https://halshs.archivesouvertes.fr/tel-01257584/document

DIRECCIÓN DE MEDICINA TRADICIONAL Y DESARROLLO INTERCULTURAL. (2017). Guía de implementación. Modelos clínicos terapéuticos y de fortalecimiento de la salud. Secretaria de salud. México. Disponible en https://www.gob.mx/salud/acciones-yprogramas/medicinas-complementarias

ESPINOSA BRITO, A. (2013). La paradoja de la salud y el modelo médico hegemónico. Revista Cubana de Salud Pública. 2013; 39(1) 1-3. Disponible en https://www.scielosp.org/pdf/rcsp/2013.v39n1/1-3/es

IGLESIAS APARICIO, P. (2003). Mujer y salud. Las Escuelas de Medicina de Mujeres. La batalla de las mujeres por el acceso al ejercicio de la Medina en el Siglo XIX. Editorial Académica Española. Disponible en https://www.academia.edu/28742084/MUJER_Y_SALUD._LAS_ESCUELAS_ DE_MEDICINA_DE_MUJERES.pdf

JOHANNESSEN, H. (1998). Una visión general de la medicina alternativa en Europa: Distribución, usos y efectos. Revista Natura Medicatrix n. 51 Octubre .1998. Disponible en https://dialnet.unirioja.es/descarga/articulo/4989315.pdf.

LAS PROVINCIAS. (2019). El cambio climático reducirá la esperanza de vida de los recién nacidos. Disponible en https://www.lasprovincias.es/sociedad/salud/cambioclimatico-esperanza-vida-recien-nacidos-20191114164019ntrc.html?ref=https\%3A\%2F\%2Fwww.google.com\%2F

MASSÉ GARCÍA, M. d. C. (2017). La mujer y el cuidado de la vida: comprensión histórica y perspectivas de futuro. Cuadernos de Bioética XXVIII 2017/3a. Disponible en http://aebioetica.org/revistas/2017/28/94/291.pdf

MOREIRA, A. (2019). Estado da arte da medicina integrativa no mundo. Revista Medicina Integrativa. Disponible en https://revistamedicinaintegrativa.com/estado-da-arteda-medicina-integrativa-no-mundo/

MUÑOZ VAN DEN EYNDE, A. (2019). La ciencia en la encrucijada: las trampas en el camino del sistema científico. 2o Informe sobre la Ciencia y la Tecnología en España. Fundación Alternativas. Disponible en https://www.fundacionalternativas.org/public/storage/laboratorio_documentos_ar chivos/b5cf15568e249cfa16b73e8bef0f1003.pdf

OFFICE FÉDÉRAL DE LA SANTÉ PUBLIQUE. (S/f). Unité de direction Assurance maladie et accidents. Médecines complémentaires dans l'assurance obligatoire des soins (AOS) /processus et critères. Disponible en https://www.admin.ch/ch/f/gg/pc/documents/2773/Medecinescomplementaires_P rocessus-et-criteres_fr.pdf 1 
ORGANIZACIÓN MUNDIAL DE LA SALUD. (2018). Contaminación atmosférica y salud infantil: prescribir aire limpio. Disponible en https://apps.who.int/iris/handle/10665/275548

PAPADAKIS, M. A.; MCPHEE, S.; RABOW, M. (2015). Diagnóstico clínico y tratamiento, 54e. McGRAW-HILL/INTERAMERICANA EDITORES, S.A. de C.V. Disponible en https://accessmedicina. mhmedical.com/book.aspx?bookid=1610

RÉ, C. (2016). Morir cuidando. Revista Rol de Enfermería 2016;39 (2): 90-97.

SCHULZ, Pablo C. (2003). Los fraudes científicos. Revista Iberoamericana de Polímeros. $\begin{array}{llll}\text { Volumen } & 4 & \text { (2). } & \text { Disponible }\end{array}$ http://www3.uah.es/vivatacademia/ficheros/n45/fraudes.pdf

TAMEZ PÉREZ, H. E. (2012). EL Abc de la Medicina científica. Mc Graw-Hill Interamericana Editores, S.A. Disponible en http://www.crids.uanl.mx/img/El\%20ABC\%20de\%20la\%20Medicina\%20Cientifica\%2 Ode\%20HE\%20Tamez\%20P\%C3\%A9rez.pdf

VÉLIZ ROJAS, L. (2017). Acompañamiento y cuidado holístico de enfermería en personas con enfermedades crónicas no adherentes al tratamiento. Revista electrónica Enfermería Actual en Costa Rica, №. 32. Disponible en https://www.scielo.sa.cr/pdf/enfermeria/n32/1409-4568-enfermeria-32-00186.pdf

* Cecilia Ré: Dra. en Pensamiento Complejo, Educación. Multiversidad Mundo Real Edgar Morin (Hermosillo, Sonora, México). Mgt. En Educación, ort. Formación a Distancia (Téluq, UQAM, Québec, Canadá). Esp. En Docencia Universitaria (UTN, San Nicolás, Argentina.). Lic. En Enfermería (UNC, Córdoba, Arg.). Tec. Univ. En Com. Soc. (UNPA, Santa Cruz, Argentina). [E-mail: cre@uarg.unpa.edu.ar] 